\title{
A Pattern Recognition Tool for Quantitative Analysis of In Planta Hyphal Growth of Powdery Mildew Fungi
}

\author{
U. Seiffert and P. Schweizer \\ Institute of Plant Genetics and Crop Plant Research, Corrensstrasse 3, D-06466 Gatersleben, Germany \\ Submitted 27 April 2005. Accepted 2 May 2005.
}

\begin{abstract}
The development of fungal pathogens can be quantified easily at the level of spore germination or penetration. However, the exact quantification of hyphal growth rates after initial, successful host invasion is much more difficult. Here, we report on the development of a new pattern recognition software (HyphArea) for automated quantitative analysis of hyphal growth rates of powdery mildew fungi on plant surfaces that usually represent highly irregular and noisy image backgrounds. By using HyphArea, we measured growth rates of colonies of the barley powdery mildew, Blumeria graminis f. sp. hordei, on susceptible and induced-resistant host plants. Hyphal growth was not influenced by the resistance state of the plants up to $48 \mathrm{~h}$ postinoculation. At later time points, growth rate increased on susceptible plants, whereas it remained restricted on induced-resistant plants. This difference in hyphal growth rate was accompanied by lack of secondary haustoria formation on induced-resistant plants, suggesting that induced resistance in barley against Blumeria graminis is caused mainly by reduced penetration rates of primary as well as secondary appressoria leading, finally, to fewer and lessdeveloped fungal colonies. No evidence was found for reduced nutrient-uptake efficiency of the primary haustoria in induced-resistant leaves, which would be expected to have resulted in reduced hyphal growth rates during the first $48 \mathrm{~h}$ of the interaction.
\end{abstract}

Additional keywords: image segmentation.

The interaction between phytopathogenic fungi and their host plants can be divided into several more or less defined stages. The first event after a fungal conidiospore has reached a plant surface is germination and formation of a germination tube. This germ tube often differentiates into an appressorium (APP) that can breach the plant cuticle and cell wall by hydrostatic, biochemical, or a combination of both forces. After successful penetration, the fungus often forms a feeding structure called a haustorium (HAU), which invaginates host plasmalemma and which is surrounded by an extrahaustorial matrix supposedly involved in nutrient transport into the fungus. HAU formation is required for hyphal growth and formation of sexual or asexual spores.

In the case of the barley powdery mildew caused by Blumeria graminis (DC.) E. O. Speer f. sp. hordei, HAU formation occurs approximately 20 to $24 \mathrm{~h}$ postinoculation (hpi),

Corresponding author: Patrick Schweizer;

E-mail: schweiz@ipk-gatersleben.de and secondary elongating hyphae (ESH) start to grow out from appressorial germ tubes at 24 to $28 \mathrm{hpi}$, as soon as the HAU is functional (Andersen and Torp 1986; Johnson et al. 1979). These ESH grow epiphytically, carry out additional penetration attempts by secondary APP, and finally lead to macroscopically visible, sporulating colonies 5 days postinoculation. For B. graminis f. sp. hordei, many data are available regarding the influence of fungal or host genotype or host treatments on conidia germination, appressorial penetration, plant papilla formation, or hypersensitive reaction of attacked host cells (Schulze-Lefert and Panstruga 2003). On the other hand, data of fungal growth rates after successful penetration are scarce, reflecting difficulties to obtain such data by quantitative microscopy. However, some reports have suggested that hyphal growth rates are influenced by host responses to the pathogen or by environmental factors (Duggal et al. 2000; Ouchi et al. 1974; Slovakova 1991; Stenzel et al. 1985; Toyoda et al. 1987). The availability of a precise tool for high-throughput screening of altered hyphal growth phenotypes would enable plant pathologists to search for new sources of plant resistance that are based on reduced HAU efficiency, which are likely to be reflected by reduced hyphal growth, rather than on reduced penetration efficiency or hypersensitive cell death. Also, late resistance responses or the impact of fungal mutations on hyphal growth rates could be precisely quantified.

Induced resistance in barley and other crop plants has been described. In the barley- $B$. graminis f. sp. hordei and riceMagnaporthe grisea pathosystems, the phenomenon of biologically induced resistance may be of practical importance (Finckh et al. 1999; Zhu et al. 2000). In both cases, cultivar mixtures carrying different resistance genes resulted in markedly reduced disease in the field, and it was speculated that this effect was due to induction of resistance by fungal races that had overcome the resistance of cultivar 1 but that were still incompatible on cultivar 2 and vice versa. Induced resistance of barley against B. graminis f. sp. hordei has been studied extensively as far as early responses of attacked cells are concerned (Kogel et al. 1994; Lyngkjaer and Carver 1999b; Schweizer et al. 1996). On the other hand, little is known about later host responses that lead to smaller colony size and, as a consequence, reduced sporulation (Ouchi et al. 1976; Stenzel et al. 1985).

Often, pattern recognition tools are applied in order to collect rather low-dimensional information from usually high-dimensional data sources. Generally, this is relevant not only for visual patterns but also for patterns in audio signals or any signal domains without a direct physical equivalence. In the case of visual patterns, other than some technical equipment to acquire the images themselves, often no further technical solutions are 
required to identify different patterns in complex and maybe distorted scenes by manual inspection. However, human inspection is subjective, rather slow, and fault-prone. This problem could be overcome by versatile computer-based (visual) pattern recognition systems. Unfortunately, such systems still are largely undeveloped. As a consequence, a large number of methods and algorithms are available in order to implement and adapt specific computerized recognition systems. In the context of the biological experiments described above, a reliable, powerful, and high-throughput system is required to automatically measure particular structures within an extensive repository of microscopic images.

Here, we report on the development of a new software (HyphArea) for automated quantitative analysis of hyphal growth rates of powdery mildew fungi. The tool was used for analysis of colony development of $B$. graminis $\mathrm{f}$. $\mathrm{sp}$. hordei on susceptible and on induced-resistant leaves of barley.

\section{RESULTS}

\section{Development of the HyphArea software.}

Automated detection and quantification of highly variable structures such as fungal colonies in front of an extremely noisy image background (i.e., a plant leaf) is a challenging task. The HyphArea software, which will be freely available for nonprofit-oriented research, carries out a series of tasks in order to automatically quantify the surface of powdery mildew colonies (Fig. 1). First, detection of fungal colonies (i.e., regions of interest) is done by applying a hierarchical set of general geometrical features. Second, after regions of interest have been found, contrast enhancement is achieved by white-and black top hat transformation. Third, median filtering is done in order to reduce noise (i.e., erratic pixels or pixel areas). Fourth, segmentation (i.e., separation of fungal hyphae from background) is achieved by applying histogram-based thresholds after color-space transformation. Fifth, image segmentation is optimized by applying morphological operators. Importantly, by combining these steps, HyphArea also can be used for quantitative analysis of growth rates of colonies feeding from single, transformed epidermal cells (Douchkov et al. 2005; Schweizer et al. 1999). Currently, HyphArea exists as fully functional, platform-independent MatLab-based implementation (Gonzalez et al. 2003). It is intended to develop a directly executable version for a number of hardware platforms in order to further support batch operation as well as to implement it on parallel hardware with improved performance for processing of large-scale image data sets. The currently available version of HyphArea will accurately detect fungal colonies from 32 hpi onward. An improved version allowing to detect even very small colonies from approximately 28 hpi onward is currently being developed.

\section{Resistance induction.}

Primary inoculation of barley with the wheat powdery mildew B. graminis (DC.) E. O. Speer f. sp. tritici induced resistance to challenge inoculation with B. graminis f. sp. hordei $24 \mathrm{~h}$ later (Table 1). These data are in agreement with earlier reports (Ouchi et al. 1974; Schweizer et al. 1996).

\section{Software-supported analysis of colony development.}

Quantitative analysis of the development of fungal colonies was carried out by using the HyphArea software. Because the thickness of fungal hyphae remains more or less constant during development of $B$. graminis f. sp. hordei on leaf surfaces, the calculated colony surface reflects total length of ESH. A comparison of ESH length between control and induced-resistant leaves was possible because primary inoculation with $B$. graminis f. sp. tritici did not completely prevent the formation of $B$. graminis f. sp. hordei colonies on induced-resistant
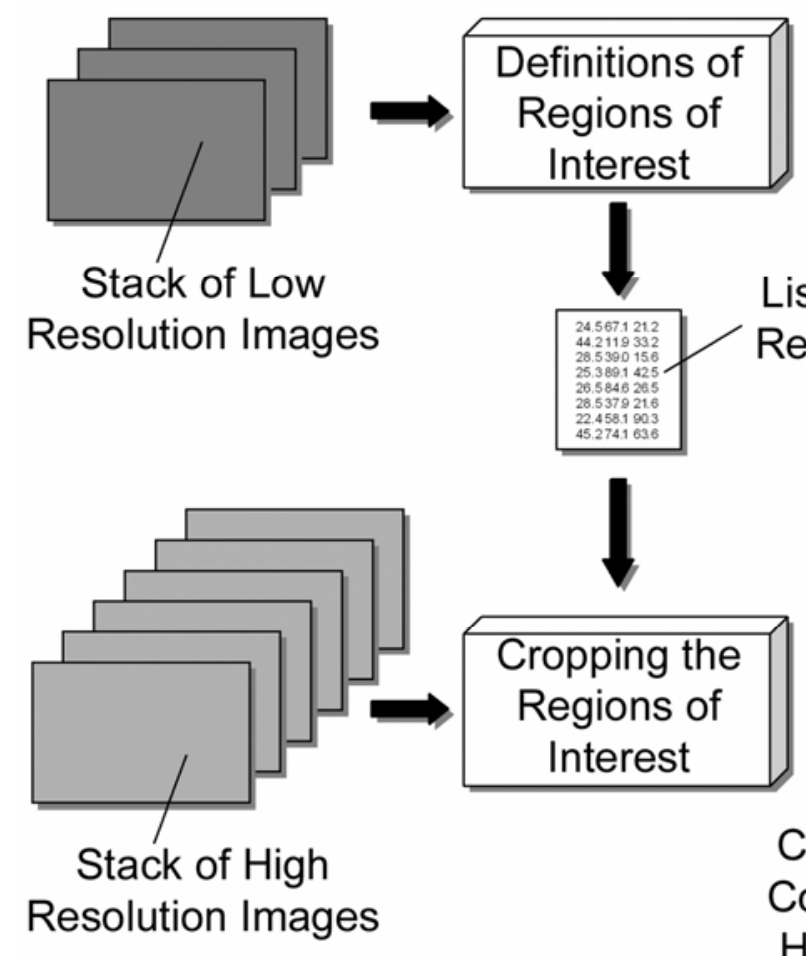
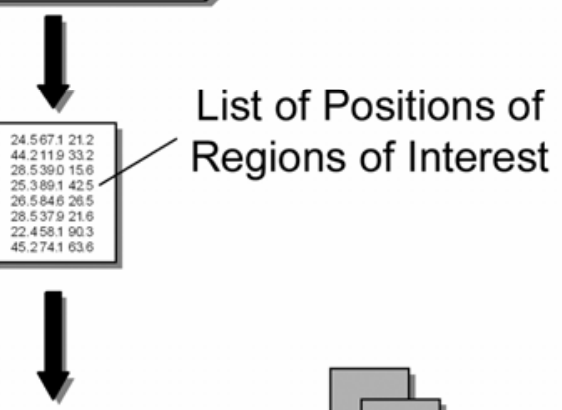
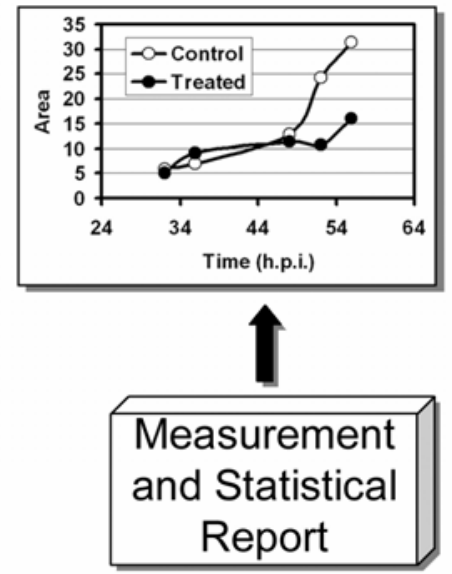

Fig. 1. Flow chart of the complete image processing system. Starting with low-resolution images, all areas that contain a single hyphal colony (region of interest) are defined. Subsequently, all of these regions are cropped from the corresponding high-resolution images. All cropped images are separately processed to segment the fungal colonies and to measure their surface. 
leaves (Table 1). ESH length increased similarly from 32 until $48 \mathrm{hpi}$ on both susceptible control leaves and induced-resistant leaves, without significant difference in the slope of growth curves (Fig. 2). From 48 hpi onward, ESH growth rate increased on susceptible control leaves, whereas it remained restricted on induced-resistant leaves. One possible explanation of this phenomenon could be the establishment, on control leaves, of secondary HAU approximately $48 \mathrm{hpi}$, which then allowed increased flow of nutrients into the growing colony. On inducedresistant leaves, however, secondary HAU formation might have been impaired.

\section{Manual analysis of haustorium formation.}

To test the hypothesis that induced resistance inhibits secondary HAU formation, HAU numbers of growing colonies were quantified manually by microscopic analysis 48 hpi (Fig. 3). The number of colonies per leaf area was reduced on inducedresistant leaves, as expected from the disease scoring data. Indeed, the pattern of HAU formation between control and induced-resistant leaves was very different. On susceptible control leaves, colonies with $1 \mathrm{HAU}$ in the first attacked cell and with at least one secondary HAU in the same cell were approximately equally distributed. By contrast, on inducedresistant leaves, the fungus almost completely failed to establish a second HAU in the successfully penetrated cell. Secondary HAU formation in neighboring cells also was less frequent in induced-resistant leaves. It also is interesting to note that the successful fungus on control leaves preferred to establish secondary HAU in the first successfully penetrated cell, perhaps due to induced accessibility of this cell (Lyngkjaer and Carver 1999b).
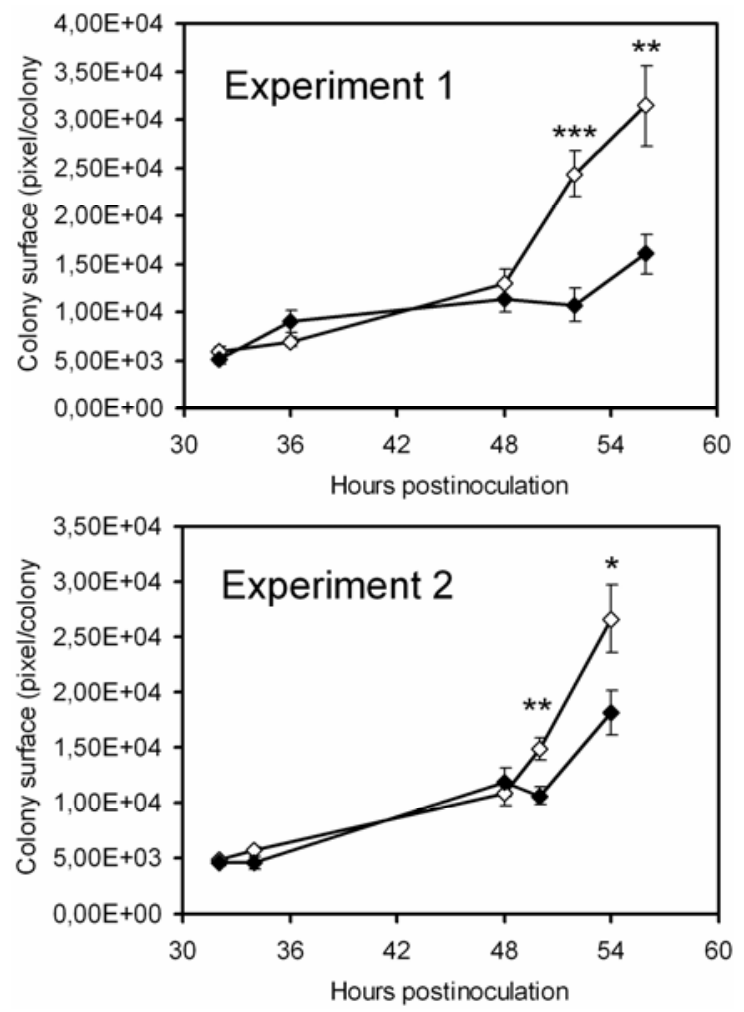

Fig. 2. Fungal growth on control and induced-resistant barley leaves. Colony surface was calculated by using the HyphArea software. Mean \pm standard error of the mean based on an average of 18 colonies per treatment and time point. The results from two independent inoculation experiments are shown. Statistical significance of differences between treatments was calculated by Student's $t$ test $\left(^{*}, * *\right.$, and $* * *=P<0.05$, 0.01 , and 0.001 , respectively.).

\section{DISCUSSION}

HyphArea allows an adequate recognition and measurement of powdery mildew colonies as long as they have not become confluent during the time course of the experiment. The morphological appearance of the fungus strongly depends on the running time of the experiment (hours postinoculation). In order to cope with these changes, all major parameters controlling the behavior and performance of the recognition system are kept variable and allow adjustment to changes over time in hyphal growth characteristics of the studied fungus. This also means that the entire system can be adapted to variable morphological features of different powdery mildew isolates, suggesting that HyphArea may also be useful for quantitative growth analysis of additional powdery mildew fungi belonging to the order Erysiphales with more than 650 known species (Schulze-Lefert and Vogel 2000).

The software tool HyphArea was tested in the barley- $B$. graminis f. sp. hordei interaction by addressing the question of whether induced resistance is based on reduced penetration rate, reduced haustorium efficiency, or both. This questions were motivated by the observation that induced resistance of barley is associated with reduced number of colonies as well as reduced ESH length (Ouchi et al. 1974; Stenzel et al. 1985). Based on the data presented in Figures 2 and 3, we suggest a model of induced resistance that is based exclusively on reduced penetration rate of primary and secondary APP, leading finally to less and smaller colonies per inoculated leaf area (Table 1). Interestingly, initially successful fungal sporelings able to form a first HAU were not able to form secondary HAU in the penetrated cell. This result suggests that induced accessibility as a result of successful host penetration is not functional in inducedresistant leaves. In contrast, induced accessibility has been shown to be effective in a genetically determined, penetrationresistant interaction (Lyngkjaer and Carver 1999a). Therefore, one may speculate that successfully penetrated cells of inducedresistant leaves obtain signals from neighboring, nonpenetrated cells, allowing them to re-establish the induced-resistant state and to overcome HAU-induced accessibility. These signals might have accumulated during $24 \mathrm{~h}$ between inducing and challenge inoculation.

In conclusion, the developed pattern recognition tool will allow users to search in a precise and targeted manner for new types of disease resistance that are based on reduced fungal nutrition (reduced HAU efficiency) or late (partial) host cell death. Gene bank collections like the one at the Institute of Plant Genetics and Crop Plant Research (IPK) represent an excellent resource for the identification of new resistance types of crop plants against the important group of powdery mildew pathogens. Another potential field of application of HyphArea is high-throughput single-cell RNAi to address gene function in the attacked model plant genotypes (Douchkov et al. 2005).

Table 1. Induced resistance of barley by primary inoculation with Blumeria graminis f. sp. tritici

\begin{tabular}{lcc}
\hline & \multicolumn{2}{c}{ Number of leaves } \\
\cline { 2 - 3 } Class of disease scoring $^{\mathbf{a}}$ & Control & Induced \\
\hline 0 & 0 & 1 \\
1 & 0 & 5 \\
2 & 7 & 9 \\
3 & 13 & 5 \\
$P\left(\chi^{2}\right)$ & $\ldots$ & 0.0202 \\
${\text { Infected leaf area }(\%)^{\mathrm{b}}}^{\mathrm{b}}$ & 62.1 & 39.7 \\
\hline
\end{tabular}

${ }^{a}$ Disease was scored 8 days postinoculation. Class $0=0$ to $5 \%$ leaf area covered with mildew pustules, class $1=6$ to $25 \%$ covered, class $2=26$ to $50 \%$ covered, and class $3=76$ to $100 \%$ covered.

${ }^{\mathrm{b}}$ Mean infected leaf area calculated by using median values per class. 
The required, manual, or automated labeling of those fungal colonies that grow out from transformed epidermal cells would be easily possible.

\section{MATERIALS AND METHODS}

Plants and fungi.

Barley cv. Golden Promise was grown in pots of compost soil (from IPK nursery) in a growth chamber (16 h of light from metal halogen lamps, $8 \mathrm{~h}$ of dark, $70 \%$ relative humidity, $20^{\circ} \mathrm{C}$ constant temperature). B. graminis DC. Speer f. sp. hordei (isolate 4.8 carrying AvrMla9) was maintained at $22^{\circ} \mathrm{C}$ and $16 \mathrm{~h}$ if light by weekly transfer to fresh barley cv. Golden Promise. B. graminis DC. Speer f. sp. tritici em Marchal (Swiss field isolate FAL 92315) was maintained at $20^{\circ} \mathrm{C}$ and $16 \mathrm{~h}$ of light by weekly transfer to fresh wheat cv. Kanzler.

\section{Resistance induction.}

Primary leaves of 7-day-old plants were cut and placed onto $1 \%$ (wt/vol) phytoagar (Ducheva, Haarlem, The Netherlands) containing benzimidazol at $20 \mathrm{ppm}$, followed by inoculation with $B$. graminis f. sp. tritici at a spore density of 120 to 170 conidia $\mathrm{mm}^{-2}$. Inoculated as well as noninoculated control leaf segments were incubated at 18 to $20^{\circ} \mathrm{C}$ and indirect day light (not supplemented with artificial light) in a climatized room. These incubation conditions were found to be optimal for delaying leaf senescence. The primary inoculum was removed mechanically from the leaf surface 24 hpi by gently rubbing with wet cotton. Control leaf segments were treated the same way. All leaves then were challenge inoculated with $B$. graminis $\mathrm{f}$. $\mathrm{sp}$. horde $i$ at a spore density of approximately 20 conidia $\mathrm{mm}^{-2}$. At the time points indicated above in Results, leaf segments were infiltrated with $20 \%$ (vol/vol) methanol and $0.1 \%$ ( vol $/ \mathrm{vol})$ Triton $\mathrm{X}-100$ and incubated at $37^{\circ} \mathrm{C}$ overnight to enhance subsequent staining of epiphytic fungal structures by Coomassie blue R-250 as described (Schweizer et al. 1993).

\section{HAU formation.}

HAU formation was analyzed manually by light microscopy 48 hpi by using a Zeiss Axioplan 2 microscope (Carl-Zeiss Co., Jena, Germany). Mature primary and secondary HAU as well as secondary HAU primordia were counted. The criterion for a HAU to be scored as secondary was APP type (differentiated ESH and not appressorial germ tube). The leaf segments used were identical to the ones used for colony surface calculation at this time point.

\section{Definition of regions of interest.}

Each hyphal colony has to be processed as a whole; therefore, an automatic detection of regions of interest (ROI) containing one complete colony has to be performed. For that purpose, all hyphal colonies have to be found and identified at an image resolution level coarse enough to contain a sufficient part of the leaf and, on the other hand, accurate enough to provide a sufficient level of details for the following processing steps (Fig. 1). The definition of the ROIs is based on a color morphological approach (Giardina and Dougherty 1998) combined with a feature extraction and a downstream classification. Within each of the three color channels, a morphological opening and closing (see equations 3 and 4 below) is performed to distinguish the desired structures (hyphal colonies). This serves as some kind of preprocessing for the binarization being necessary to turn image areas into objects. Then, a number of features which are specific to the hyphal colonies are extracted from all considered objects found in the images. Because the colonies are characterized by a rather large variability, all template matching-based approaches, for example, had to be dropped out and only very general geometrical features could have been considered. Surprisingly, just four features turned out to be sufficient to successfully recognize more than $90 \%$ of all existing colonies. Because none of the tested feature spaces (also those with more than the described four features) led to a proper clustering of the objects, a hierarchical classification has been implemented. The features are, in detail (according to their hierarchical level within the classification), i) circularity, ii) solidity (rectangularity), iii) principal axis of the bounding box enclosing the considered object (minimum enclosing rectangle), and iv) surface area of the object.

The first feature is a shape factor specifying the ratio between the circumference $C$ of the object and its surface area A: $\operatorname{Circularity}(o)=C / A$ (equation 1$)$.

All objects falling below a given threshold are rejected and not further tested. All remaining objects are tested with the second feature, which is the ratio between the object's surface area $A$ and the surface area of the minimum enclosing rectangle $A_{\text {mer }}: \operatorname{Solidity}(o)=A / A_{\text {mer }}$ (equation 2 ).

Both equations 1 and 2 are nondimensional and thus independent of the size of the tested objects. However, in order to sort out objects being too small or too large, the size-dependent third and fourth features are used. The third one mainly eliminates large-area objects caused by unwanted infiltration of dye into the leaf. Finally, the mean value of the surface areas (feature four) of all remaining objects is utilized to detect all objects being outside the expected size band of the objects. In this way, an automatic recognition of hyphal colonies has been implemented, which is characterized by high efficiency, robustness, versatility (particularly important against the background of enormous variability of the processed colonies depending on the temporal set-up of the biological experiment), and only a low number of parameters. This results in cropped images, each containing a single hyphal colony, which are further processed.

\section{Image preprocessing.}

The images were acquired using a Zeiss Axioplan2 light microscope with 50-fold magnification. The initial image resolution was 2,600 by 2,060 pixels with a native color depth of 3 by 14 bits/pixel. For the described analysis, a color depth of 8 bits/color channel (red, green, and blue) turned out to be sufficient and particularly more efficiently manageable. A number of image preprocessing steps, such as contrast enhancement as

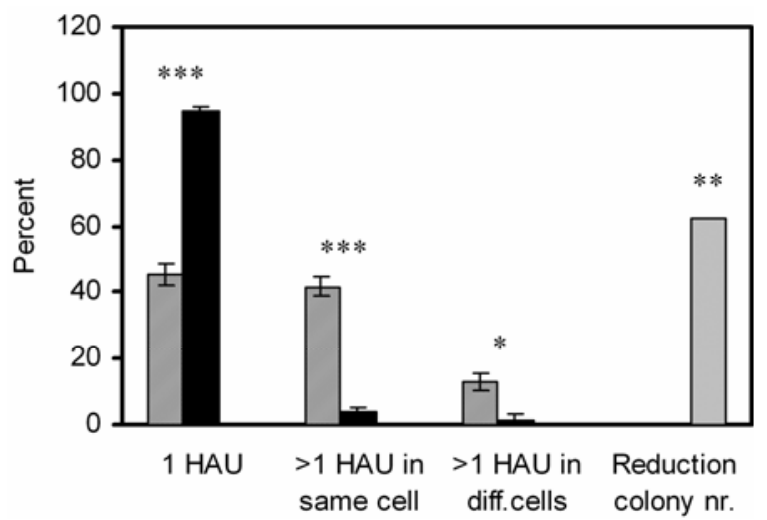

Fig. 3. Haustoria formation of developing colonies on control or inducedresistant barley leaves. Haustoria were counted by manual light microscopy at $400 \times$ magnification $48 \mathrm{~h}$ postinoculation. Striped columns, control; black columns, induced resistant. Data are based on 600 analyzed fungal colonies in two independent experiments. Mean \pm standard error of the mean of 14 leaf segments. Statistical significance of differences between treatments was calculated by Student's $t$ test $(*, * *$, and $* * *=P<$ $0.01,0.001$, and 0.0001 , respectively). 
well as median filtering, were applied to the raw image material (Fig. 4A). At the inoculum density chosen $\left(20\right.$ conidia $\left.\mathrm{mm}^{-2}\right)$, approximately 50 colonies were visible on control leaves per initial image.

\section{Contrast enhancement.}

Contrast enhancement (Gonzalez and Woods 2002) was used to emphasize particular image properties. Generally, there exist pixel-based, neighborhood-based, and transformationbased methods. The class of the top hat transformations, representing neighborhood-based transformations, proved to be most advantageous. A neighborhood-based operator can be obtained by applying both the white and black top hat transformation to the images. The white top hat transformation (Fig. 4B) is defined as difference between the original image $f$ and its opening $\gamma$. White top hat $(f)=f-\varkappa(f)$ (equation 3 ).

The black top hat transformation (Fig. 4C) is defined as difference between the closing $\phi$ of the original image $f$ and the original image $f$ itself: Black top hat $(f)=\phi(f)-f$ (equation 4$)$.

Now the neighborhood-based image operator $\kappa$ (Fig. 4D) can be computed as: $\kappa=f+$ White top hat $(f)-$ Black top hat $(f)$ (equation 5).
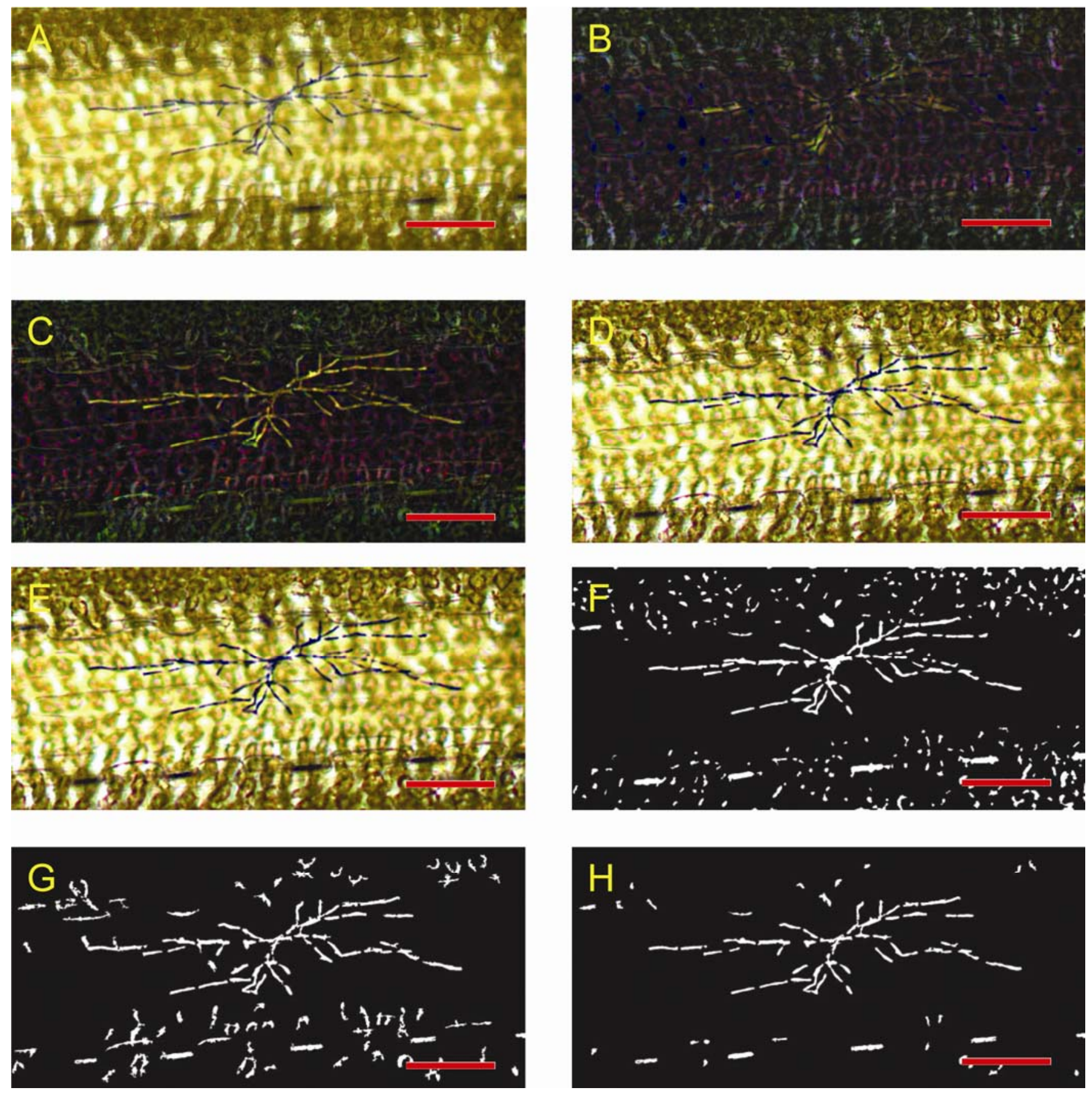

Fig. 4. Image processing chain. Showcase of an original image $f$ containing a part of a barley leaf and one colony of Blumeria graminis f. sp. hordei. A, Original sample image. Based on this sample image the results are shown for $\mathbf{B}$, the white top hat transformation, $\mathbf{C}$, the black top hat transformation, and $\mathbf{D}$, the contrast enhancement $\kappa$, all with rectangular structural elements according to equations 3,4 , and 5 . E, Result of the median filtering of the previously contrast enhanced image as shown in panel D. F, Sample image of panel E now segmented with adaptive thresholds derived from the YCbCr histograms of the original sample image. G, Resulting sample image when applying the image operator $\rho$ (equation 6) directly to image A. H, Final black-and-white image resulting from logic combination of the two independently segmented images of $\mathrm{F}$ and $\mathrm{G}$. A through $\mathrm{H}$, Scale bar corresponds to $100 \mu \mathrm{m}$. 
By choosing an appropriate structural element of the opening and closing operations, the impact on those image structures to be contrast enhanced can be tuned. All structures of the original image not matching the selected structural element gradually are suppressed by the top hat transformation. The major growth direction of the hyphae is along the abscissa; therefore, a rectangular structural element in landscape orientation seems to be appropriate. This also partly compensates for the apparent gaps of the hyphae caused by unstained septa.

\section{Median filtering.}

When looking at the details of the original image (Fig. 4A), it clearly can be seen that there is a particular level of noise, which is even boosted by the contrast enhancement (Fig. 4D). In order to reduce this noise without changing the remaining information of the images (to be analyzed later), a median filtering was performed (Fig. 4E). Here, the gray values of all pixels belonging to a fixed neighborhood of a considered pixel were ordered and, subsequently, the median was assigned to the considered pixel. By applying this nonlinear filter, erratic pixels or pixel areas (depending on the size of the neighborhood) are eliminated. Nevertheless, the appearance of the hyphae is not significantly affected by this operation. Similar to the contrast enhancement described above, the impact of this filter on the images can be tuned by a number of parameters. Generally speaking, the larger the filter mask, the more the image gets smoothed, and vice versa. In order to retain the positive effects of the contrast enhancement, the size of the filter mask should stay rather small.

\section{Segmentation and histogram-based thresholds.}

As in many image-processing tasks, proper segmentation is one of the most crucial parts. Here, an algorithm distinguishing between different image regions, namely hyphae and background, is required. As a criterion of this discrimination, several properties of all image regions (strictly speaking, of all image pixels) can be utilized. Commonly, image segmentation can efficiently be done by defining several thresholds based on histogram analysis. Unfortunately the original red-green-blue (RGB) color space is not suitable to automatically derive significant thresholds from the histograms (Fig. 5A). Obviously, the RGB color space is not fit for a discrimination between hyphae and background.

Since the human eye is well able to recognize hyphal colonies while the RGB color space is the native (coming from the digital camera) but just one possible representation of the images, there must exist a more suitable image representation. Consequently, a color space transformation can be used. A very common candidate (Ihlow and Seiffert 2003) in this context is the hue-saturation-value (HSV) color space representing each image pixel as a triplet of its hue, saturation, and value fraction. Although the histograms in HSV color space were found to be quite meaningful, the YCbCr (Gonzalez and Woods 2002) color space performed best. In this format, luminance information is stored as a single component $(\mathrm{Y})$, and chrominance information is stored as two color-difference components $(\mathrm{Cb}$ and $\mathrm{Cr}$ ). $\mathrm{Cb}$ represents the difference between the blue component and a reference value. Cr represents the difference between the red component and a reference value. The corresponding histograms are shown in Figure 5B.

The sample image of Figure 4A, now segmented with adaptive thresholds derived from the $\mathrm{YCbCr}$ histograms, is shown in Figure 4F. Although the fungal colony can be seen clearly, there are still many erroneously segmented areas. Because the segmentation is based on rather simple thresholds (regardless of the color space in which the image actually is represented), all areas of the image similar enough, in terms of color and contrast, to fall between the defined thresholds are determined as the target structure; namely, hyphae.

\section{Morphological operators.}

Alternatively, some morphological filter operations can be directly used for segmentation (Dougherty and Lotufo 2003). This step is motivated by the positive results of the white and black top hat operations applied to the original images, as shown in Figs. 4B and 4C. By subtracting the White top hat $(f)$ (equation 3) from the Black top hat (f) (equation 4), a new image operator $\rho$ can be defined: $\rho=$ Black top hat $(f)-$ White top $\operatorname{hat}(f)=\phi(f)+\chi(f)-2 f($ equation 6$)$

The resulting segmented sample image is shown in Figure 4G. Compared with Figure 4F, it clearly can be seen that many small regions not belonging to the considered hyphal colony are not present anymore. However, there still exist erroneously segmented regions.

\section{Combining both methods.}

Obviously, neither of the two described operations leads to satisfactory results. However, by a logic operation of both segmented images, the histogram threshold-based one (Fig. 4F) and the morphological filter-based one (Fig. 4G), a further improved segmentation can be achieved (Fig. $4 \mathrm{H})$.
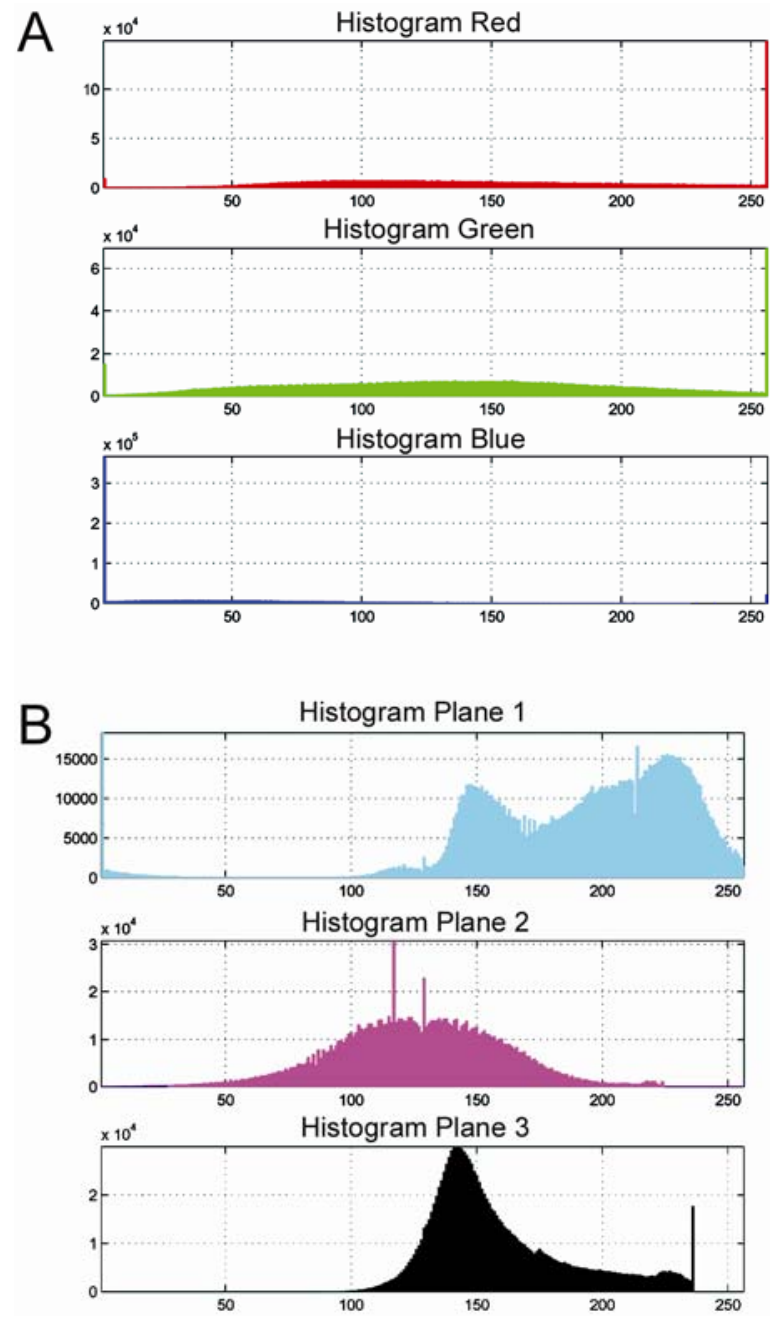

Fig. 5. Histograms of the three color channels of the sample image of Figure 4A. A, Image in the original red-green-blue color space. B, Histogram of a $\mathrm{YCbCr}$ color space representation of the same sample image. Compared with A, the shape of the single histograms are much more structured and can be used to define discrimination thresholds. 


\section{ACKNOWLEDGMENTS}

This work was supported by the Institute of Plant Genetics and Crop Plant Research, a member of the Leibniz Society (to P. Schweizer), and by grant number 0312706A of the German Federal Ministry of Education and Research (to U. Seiffert). The excellent technical assistance of S. Gentz, M. Knauft, and S. Lück is acknowledged. We appreciate the distinguished work of C. Schulze for implementing and testing the pattern recognition tool.

\section{LITERATURE}

Andersen, J. B., and Torp, J. 1986. Quantitative analysis of the early powdery mildew infection stages on resistant barley genotypes. J. Phytopathol. Phytopathol. Z. 115:173-186.

Douchkov, D., Nowara, D., Zierold, U., and Schweizer, P. 2005. A highthroughput gene silencing system for the functional assessment of defense-related genes in barley epidermal cells. Mol. Plant-Microbe Interact. 18:755-761.

Dougherty, E. R., and Lotufo, R. A. 2003. Hands-on Morphological Image Processing. SPIE International Society for Optical Engineering, Bellingham, WA, U.S.A.

Duggal, V., Jellis, G. J., Hollins, T. W., and Stratford, R. 2000. Resistance to powdery mildew in mutant lines of the susceptible wheat cultivar Hobbit 'sib'. Plant Pathol. 49:468-476.

Finckh, M. R., Gacek, E. S., Czembor, H. J., and Wolfe, M. S. 1999. Host frequency and density effects on powdery mildew and yield in mixtures of barley cultivars. Plant Pathol. 48:807-816.

Giardina, C. R., and Dougherty, E. R. 1998. Morphological Methods in Image and Signal Processing. Prentice Hall, Upper Saddle River, NJ, U.S.A.

Gonzalez, R. C., and Woods, R. E. 2002. Digital Image Processing, 2nd ed. Prentice Hall, Upper Saddle River, NJ, U.S.A.

Gonzalez, R. C., Woods, R. E., and Eddins, S. L. 2003. Digital Image Processing Using MATLAB. Prentice Hall, Upper Saddle River, NJ, U.S.A.

Ihlow, A., and Seiffert, U. 2003. Microscope color image segmentation for resistance analysis of barley cells against powdery mildew. Bioinformatics Center Gatersleben-Halle, Germany. http://pgrc-16.ipk-gatersleben.de/ $\sim$ seiffert/publications/Ihlow_WSFB03.pdf. Published online.

Johnson, L. E. B., Bushnell, W. R., and Zeyen, R. J. 1979. Binary pathways for analysis of primary infection and host response in populations of powdery mildew fungi. Can. J. Bot. 57:497-511.

Kogel, K. H., Beckhove, U., Dreschers, J., Munch, S., and Romme, Y. 1994. Acquired-resistance in barley-The resistance mechanism induced by 2,6-dichloroisonicotinic acid is a phenocopy of a genetically based mechanism governing race-specific powdery mildew resistance. Plant Physiol. 106:1269-1277.
Lyngkjaer, H. F., and Carver, T. L. W. 1999a. Modification of mlo5 resistance to Blumeria graminis attack in barley as a consequence of induced accessibility and inaccessibility. Physiol. Mol. Plant Pathol. 55:163174.

Lyngkjaer, M. F., and Carver, T. L. W. 1999b. Induced accessibility and inaccessibility to Blumeria graminis f. sp hordei in barley epidermal cells attacked by a compatible isolate. Physiol. Mol. Plant Pathol. 55:151-162.

Ouchi, S., Oku, H., Hibino, C., and Aldyama, I. 1974. Induction of accessibility and resistance in leaves of barley by some races of Erysiphe graminis. Phytopathol. Z. 79:24-34.

Ouchi, S., Oku, H., and Hibino, C. 1976. Localization of induced resistance and susceptibility in barley leaves inoculated with powdery mildew fungus. Phytopathology 66:901-905.

Schulze-Lefert, P., and Panstruga, R. 2003. Establishment of biotrophy by parasitic fungi and reprogramming of host cells for disease resistance. Annu. Rev. Phytopathol. 41:641-667.

Schulze-Lefert, P., and Vogel, J. 2000. Closing the ranks to attack by powdery mildew. Trends Plant Sci. 5:343-348.

Schweizer, P., Gees, R., and Mosinger, E. 1993. Effect of jasmonic acid on the interaction of barley Hordeum vulgare L. with the powdery mildew Erysiphe graminis f. sp. hordei. Plant Physiol. 102:503-511.

Schweizer, P., Jeanguenat, A., Whitacre, D., Metraux, J. P., and Mosinger, E. 1996. Induction of resistance in barley against Erysiphe graminis f. sp. hordei by free cutin monomers. Physiol. Mol. Plant Pathol. 49:103120.

Schweizer, P., Pokorny, J., Abderhalden, O., and Dudler, R. 1999. A transient assay system for the functional assessment of defense-related genes in wheat. Mol. Plant-Microbe Interact. 12:647-654.

Slovakova, L. 1991. Induced resistance of barley plants against powdery mildew (Erysiphe graminis f. sp. hordei Marchal). 1. Influence of inducers on primary infection. Biologia 46:737-744.

Stenzel, K., Steiner, U., and Schonbeck, F. 1985. Effect of induced resistance on the efficiency of powdery mildew haustoria in wheat and barley. Physiol. Plant Pathol. 27:357-367.

Toyoda, H., Matsuda, Y., Shoji, R., and Ouchi, S. 1987. A microinjection technique for conidia of Erysiphe graminis f. sp. hordei. Phytopathology 77:815-818.

Zhu, Y. Y., Chen, H. R., Fan, J. H., Wang, Y. Y., Li, Y., Chen, J. B., Fan, J. X., Yang, S. S., Hu, L. P., Leung, H., Mew, T. W., Teng, P. S., Wang, Z. H., and Mundt, C. C. 2000. Genetic diversity and disease control in rice. Nature 406:718-722.

\section{AUTHOR-RECOMMENDED INTERNET RESOURCES}

HyphArea software: hypharea.ipk-gatersleben.de The MathWorks MatLab \& Simulink webpage: www.mathworks.com 